\title{
Frequency of broad-spectrum beta-lactamase gene and evaluation of antimicrobial effect of Teucrium polium extract and essential oil in clinical isolates of Klebsiella pneumonia
}

\author{
Roghayeh Gholizadeh Doran Mahalleh ${ }^{1 *(\mathbb{}}$, Farokh Rokhbakhsh Zamein ${ }^{\circledR}{ }^{\circledR}$, Javad Dahmardeh ${ }^{(\circledR}$ \\ 'Department of Laboratory Sciences, Zahedan Branch, Islamic Azad University, Zahedan, Iran \\ ${ }^{2}$ Department of Microbiology, Kerman Branch, Islamic Azad University, Kerman, Iran \\ ${ }^{3}$ Education Secretary, Mirjaveh County, Iran
}

*Correspondence to

Roghayeh Gholizadeh

Doran Mahalleh, Email:

De.gholizadeh@gmail.com

Received 20 Aug. 2019 Accepted 21 Nov. 2019 Published online 10 Jan. 2019

Keywords: Antibacterial, Drug resistance, Klebsiella pneumoniae, Teucrium polium

\section{Abstract}

Introduction: $\beta$-Lactamase enzymes are one of the most important factors in generating antibiotic resistance among gram-negative bacteria. Klebsiella pneumoniae is an opportunistic pathogen from the Enterobacteriaceae family, which plays a major role in the development of hospital infections and people with underlying diseases. Objectives: This study was aimed to investigate the antimicrobial effect of the essential oil of Teucrium polium on strains containing antibiotic resistant genes in clinical isolates of Klebsiella pneumoniae in Zahedan.

Materials and Methods: To identify chemical compounds and to investigate the antibacterial effects of T. polium extract, the leaves of this plant were collected from their natural habitat in May 2018 at full flowering stage. Extraction was carried out by water distillation method. Antibacterial activity of the extract of this plant and the least inhibitory concentration of growth on the resistant strains of Klebsiella pneumoniae was performed.

Results: The essential oil yield of T. polium was $75 \%$. Of the 29 compounds identified in the essential oil of the $T$. polium, the combination of $\alpha$-pinene with $12.52 \%$, linalool was the highest in the essential oil of $10.63 \%$. Of the 120 isolates of $K$. pneumoniae, 52 isolates in the initial screening were positive for ESBL production, of which 39 isolates (32.5\%) were positive in the phenotypic confirmatory assay. Around 11 isolates (10.8\%) had ampC genes. Based on the results of polymerase chain reaction (PCR), $76.9 \%, 12.8 \%, 30.8 \%, 28.2 \%, 25.7 \%$ and $56.4 \%$ of the isolates were carriers of FOX, EBC, ACC, DHA, CIT genes and MOX, respectively.

Conclusion: The results of antimicrobial resistance study of isolates showed that the highest resistance to erythromycin antibiotics $(92.5 \%)$, cefotaxime $(38.4 \%)$ and ceftriaxone $(35.9 \%)$, respectively, while the highest susceptibility was observed in colistin antibiotics $(98.3 \%)$, imipenem $(90 \%)$ and amikacin $(88.3 \%)$, respectively. The essential oil of the T. polium had a significant antibacterial effect. Due to the high percentage of high concentrations of $\alpha$-pinene and linalool in the essential oil of the T. polium, in such a way that has antibacterial properties, T. polium can be used to cope with certain pathogenic bacteria.

\begin{abstract}
Citation: Gholizadeh Doran Mahalleh R, Rokhbakhsh Zamein F, Dahmardeh J. Frequency of broad-spectrum beta-lactamase gene and evaluation of antimicrobial effect of Teucrium polium extract and essential oil in clinical isolates of Klebsiella pneumonia. Immunopathol Persa. 2020;6(1):e09. DOI: 10.15171 ipp.2020.09
\end{abstract}

\section{Introduction}

Klebsiella pneumoniae is a gram-negative pathogen of opportunistic Enterobacteriaceae family that plays a major role in the development of various hospital infections such as pneumonia, sepsis, urinary tract infections, and intra-abdominal infections in hospitalized patients as well as patients with underlying diseases (1). $\quad \beta$-Lactam antimicrobial agents are currently the most commonly used treatment for bacterial infections. The widespread use of $\beta$-lactam antimicrobial agents may lead to the resistance to bacterial beta-lactam antibiotics worldwide.

AmpC plasmids enzymes are divided into six families of MOX, FOX, CIT, DHA, EBC and ACC. A small change in the sequence of

\begin{abstract}
Key point
The essential oil of the Teucrium polium had a noticeable antibacterial effect due to the high concentration of $\alpha$-pinene and linalool.
\end{abstract}

their amino acids leads to different varieties among the family (2). Over the decades after the discovery of antibiotics, their uncontrolled administration to treat bacterial infections has led to the selection and spread of resistant strains of bacteria, hence bacterial resistance to antibiotics has now become a global dilemma. The major consequence of this misguided strategy is to replace and create clones of resistant strains of bacteria instead of susceptible strains.

\footnotetext{
Copyright (C) 2020 The Author(s); Published by Nickan Research Institute. This is an open-access article distributed under the terms of the Creative Commons Attribution License (http://creativecommons.org/licenses/by/4.0), which permits unrestricted use, distribution, and reproduction in any medium, provided the original work is properly cited.
} 
Teucrium polium is herbaceous, persistent, and ramose with 10 to $35 \mathrm{~cm}$ height and has a white cotton appearance, usually in the arid, rocky, and sandy beaches of the world. Its leaves are narrow, long and covered with cotton flakes on both sides of the surface. The use of it is effective in the headache, weakness of the digestive system, urinary tract disorders, and delayed or absent menstruation due to general weakness. It also has anticonvulsant effects. This bitter plant in Baluchistan (Iran) and India is used to relieve the pain of the heart, as well as antipyretic and is consumed by the boiling water in the water and soaked or caught in the water in cases of colds. In Zahedan, people also use it to relieve the discomfort of the digestive system and the heart. This plant contains a-pinene and linalool, which enhances the probability of bacterial sensitivity. So far, studies have been done on its antibiotic properties on other species (3).

\section{Objectives}

The purpose of this study was to determine the antibiotic resistance pattern of $K$. pneumoniae strains isolated from clinical specimens of patients in educational hospitals of Zahedan, studying the frequency of AmpC $\beta$-lactamase genes in clinical isolates of $K$. pneumoniae, and comparing the therapeutic properties of T. polium with modern antibiotic therapy under laboratory conditions. Considering the creation of new gaps between conventional medicine and molecular medicine in the present age, antibiotic therapy can be selected by identifying the resistance gene in the bacteria in the shortest possible time based on the molecular analysis of the polymerase chain reaction (PCR). Introducing the results of this study will help the medical community to select the type and dose of medicine appropriately (4). If the results of infection suppression with T. polium to be positive, it can be helpful to identify and isolate the effective substance for introduction into the pharmaceutical industry.

\section{Methods and Materials}

This was a descriptive-analytical and laboratory study. K. pneumoniae samples were included urine, blood and respiratory tract secretions samples from April to September of 2018, around 230 samples of clinical isolates of $K$. pneumoniae from microbiology laboratories of Zahedan hospitals were gathered. After sample collection, suspected samples were transferred to the microbiology laboratory of the infectious disease research venter of Bu Ali hospital of Zahedan. All patient characteristics including the patient's name, hospitalization ward, sampling site, and other required specifications were recorded. Then in the laboratory, the collected samples were confirmed by standard and differential microbiological methods. Finally, 120 isolates were identified as K. pneumoniae.

The samples were inoculated on blood and MacConkey agar culture and incubated at $37^{\circ} \mathrm{C}$. After $24 \mathrm{~h}$ incubation, the first clone was extracted, the slide was prepared and the germ staining was performed. The color of the colony was examined either on the face or on color on the MacConkey agar. Then catalase and oxidase tests were performed on single colonies (5). The following biochemical tests were performed for the final diagnosis of bacteria using the standard table; 1) antibacterial culture in a TSI agar, 2) bacterial culture of the bacteria in Simmons Citrate Agar, 3) culture of the bacteria in the SIM (Sulfide, Indole, Motility), 4) endodontic test using Kovacs, 5) culture of bacteria in MR-VP liquid, 6) culture of bacteria in urea agar, 7) culture of bacteria in lysine-decarboxylase, 8) culture of bacteria in ornithine decarboxylase, 9) culture of bacteria in arginine dihydrolase and 9) investigation of fermentation of sugars.

The leaves of the T. polium from their natural habitat were collected from the villages of Sistan and Baluchistan province in April of 2018, and after identification, they were cleaned and washed and in shadow conditions due to hydrolysis of the compounds. Then, the plants were dried at ambient temperature and then $150 \mathrm{~g}$ of crushed samples were extracted by water distillation using clevenger for three hours. Then, the extract was injected into a mass spectrometer (GC/MS) coupled with the mass spectrometry and corresponding chromatograms (3). Antibiotic resistance and susceptibility patterns for 120 clinical isolates of $K$. pneumonia by disc diffusion method with antibiotic discs were as following; ceftriaxone (30 $\mu \mathrm{g})$, ceftazidime $(30 \mu \mathrm{g})$, cefotaxime $(30 \mu \mathrm{g})$, ciprofloxacin $(5 \mu \mathrm{g})$, gentamicin $(10 \mu \mathrm{g})$, amikacin $(30 \mu \mathrm{g})$, imipenem $(10 \mu \mathrm{g})$, chloramphenicol $(30 \mu \mathrm{g})$, colistin $(10 \mu \mathrm{g})$ and erythromycin $(15 \mu \mathrm{g})$ (prepared by MAST UK). In accordance with the Clinical Laboratory Standard Institute (CLSI) guidelines, the disk propagation method was used (6).

\section{Disk diffusion}

The microbial suspension prepared with dilution of interest was applied to the surface of the agar (next to the flame). Rotational rotations were done in the right and left directions to be uniformly distributed at the agar surface. After five minutes, the plates were placed in a slope and the microbial suspension was removed using the remaining sampler. Then, using sterile pins, the antibiotics were placed on the surface with proper spacing from each other and from the plate wall. Plates were placed at $37^{\circ} \mathrm{C}$ for 18 hours (7).

\section{Result of disc diffusion}

After 18 hours' incubation at $37^{\circ} \mathrm{C}$, using a ruler under the light bulb, the diameter of the inhibition zone around the antibiotic discs was measured and evaluated using the CLSI instruction. The specimens were resistant, intermediate and sensitive. In each series, the standard strain of E. coli ATCC 25922 was used to control the quality of the discs (Figure 1) (8). 


\section{Ethical issues}

The research followed the tenets of the Declaration of Helsinki. All study protocols were approved by the institutional ethical committee at Zahedan University (Ref $\# 1099706040002$ ). Also, they were in accordance with the guidelines of the National Health Institute (NIH1978) and Medical Research Council.

\section{Data analysis}

Data were analyzed using descriptive analyses as frequency and percentage. The results were reported as table and graph.

\section{Results}

Clinical samples were collected from different parts of the hospital including 75 isolates (62.5\%) from outpatients, 23 isolates (19.2\%) from intensive care unit (ICU), 8 isolates (6.7\%) from emergency department, 6 isolates (5\%) from infectious ward, 3 isolates (2.5\%) from the internal section, 4 isolates (3.3\%) from the women's department and 1 isolate $(0.8 \%)$ from the pediatric ward. Of the 120 samples of K. pneumoniae, 73 samples $(60.8 \%)$ were related to the female population and 47 samples $(39.2 \%)$ were related to the male population.

All strains of $K$. pneumoniae were analyzed after definitive isolation and diagnosis for disk diffusion test. The diameter of the inhibition zone around the discs was measured using a millimeter ruler and was evaluated in accordance with the CLSI 2017 standard table. The

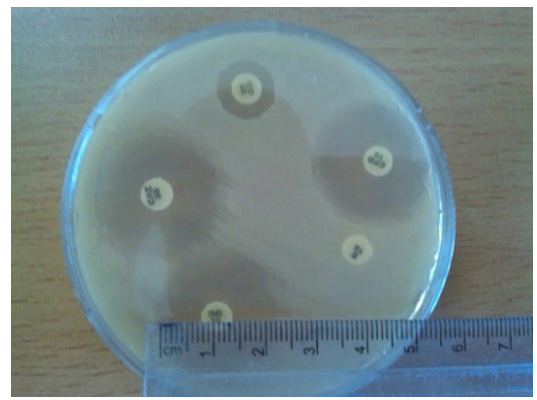

Figure 1. The result of disc diffusion samples were resistant (R), intermediate (I) and sensitive (S) (Table 1) (6).

The results showed that the yield of T. polium collected from Sistan and Baluchistan province was $0.75 \%$. Of the 28 compounds identified in the T. polium extract with $99.75 \%$, a-pinene compounds with $12.52 \%$, Linalool with $10.63 \%$, and caryophyllene oxide with $9.69 \%$ have the highest percentage of extract. The extract of this plant on the gram-negative bacteria of $K$. pneumoniae has a diameter of $15 \mathrm{~mm}$ growth inhibitory effect. As shown in Figure 2 , based on the results of PCR, in Klebsiella pneumoniae isolates 30 (76.9\%), 5 (12.8\%), 12 (30.8\%), 11 (28.2\%), 10 (25.7\%), $22(56.4 \%)$ of the isolates were carriers of FOX, EBC, ACC, DHA, CIT genes and MOX, respectively.

\section{Discussion}

Medicinal plants such as T. polium L. are one of the great important plants in the field of traditional medicine and modern medicine, with important pharmaceutical and nutritional compounds and antibacterial effects in botanical matters. (9). The results of T. polium analysis

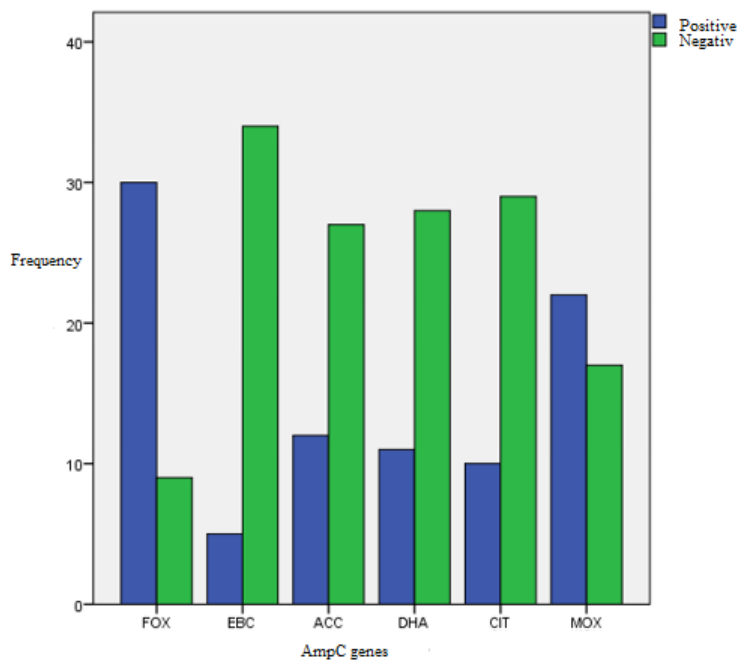

Figure 2. Frequency of genes producing broad-spectrum betalactamase enzymes in Klebsiella pneumoniae isolates.

Table 1. Frequency distribution of resistance pattern and antibiotic senility in Klebsiella pneumoniae strains isolated from educational hospitals in Zahedan according to the type of used antibiotics

\begin{tabular}{|c|c|c|c|c|c|c|c|c|}
\hline \multirow{2}{*}{ Antibiotic type } & \multirow{2}{*}{$\begin{array}{l}\text { Abbreviated code for } \\
\text { antibiotics }\end{array}$} & \multirow{2}{*}{ Disc concentration } & \multicolumn{2}{|c|}{ Resistant } & \multicolumn{2}{|c|}{ Intermediate } & \multicolumn{2}{|c|}{ Sensitive } \\
\hline & & & No. & $\%$ & No. & $\%$ & No. & $\%$ \\
\hline Chloramphenicol & $\mathrm{C}$ & $30 \mu \mathrm{g}$ & 18 & 15 & 0 & 0 & 102 & 85 \\
\hline Ceftazidime & CAZ & $30 \mu \mathrm{g}$ & 31 & 25.8 & 7 & 5.8 & 82 & 68.4 \\
\hline Ciprofloxacin & CIP & $5 \mu g$ & 28 & 23.4 & 6 & 5 & 86 & 71.6 \\
\hline Ceftriaxone & $\mathrm{CRO}$ & $30 \mu \mathrm{g}$ & 43 & 35.9 & 2 & 1.6 & 75 & 62.5 \\
\hline Cefotaxime & CTX & $30 \mu \mathrm{g}$ & 46 & 38.4 & 2 & 1.6 & 72 & 60 \\
\hline Gentamicin & GM & $10 \mu \mathrm{g}$ & 39 & 32.5 & 3 & 2.5 & 78 & 65 \\
\hline Amikacin & AK & $30 \mu g$ & 10 & 8.3 & 4 & 3.4 & 106 & 88.3 \\
\hline Imipenem & IMI & $10 \mu \mathrm{g}$ & 9 & 7.5 & 3 & 2.5 & 108 & 90 \\
\hline Colistin & $\mathrm{CO}$ & $10 \mu \mathrm{g}$ & 2 & 1.7 & 0 & 0 & 118 & 98.3 \\
\hline
\end{tabular}


collected from Sistan and Baluchistan province showed that the extract yield of this species was $75 \%$, which was less efficient than the study of Mohammad et al, that was carried out by steam distillation (3). The investigations showed that the total of 29 compounds identified in the T. polium extract with $99.75 \%$, a-pinene compounds with $12.52 \%$, Linalool with $10.63 \%$, and caryophyllene oxide with $9.69 \%$ had the highest percentage which had similarities and differences in comparison with other researchers.

In Iran and other parts of the world, a lot of research has been carried out on the different species of Teucrium, including the identification of T. stocksianum compounds by Jaimand et al that reported camphen (20.6\%), a-cadinol (19.7\%), myrcene (10.2\%) and carvacrol (9.9\%) as the main components of this herb (10). In another study, T. flavum was shown to be the most active ingredient in $\alpha$-caryophyllene (30.7\%), germacrene (21.3\%) a-humulenea (8.8\%) (9). T. orientale. L. subsp orientale has been reported with caryophyllene (33.5\%), linalool (17.0\%) and $ß$-caryophyllene (9.3\%) (11). In the study of $T$. persicum, the compounds of caryophyllene oxide (10.6\%), a-pinene (9.4\%), linalool - $\alpha$ (7.8\%), cadinene (7.4\%), elemol (9 / 6\%), $\alpha$-cadinol (5.5\%) had the highest levels (12). In the study on T. orientale.L. subsp taylori of Lorestan province of Iran, linalool (28.66\%), caryophyllene oxide (15.62\%), 3-octanol (5.55\%), B-pinene (8.75\%) and ß-caryophyllene (7.33\%) were identified as the major combinations (13). In the study of the Teucrium species in Italy, the T. fruticans species had major components including $\beta$-pinene $(21.0 \%)$, germacrene $\mathrm{D}(18.1 \%)$, $\beta$-myrcene (13.0\%) and $\beta$-caryophyllene (12.0\%) (14). In another study, the main component of $T$. polium subsp. capitatum were $\alpha$-pinene (28.8\%), $ß$-pinene (7.2\%) and p-cymene $(0.07 \%)$ (15). Accordingly, in a study in Jordan, T. polium showed the highest levels of 8-cedern-13-ol (24.8\%), ß-caryophyllene (7.8\%), germacrene D (6.8\%) and sabinene $(5.2 \%)(16,17)$.

In this study, the combination of a-pinene with $12.52 \%$, linalool with $10.63 \%$, and caryophyllene oxide with $9.69 \%$ constitute the highest percentage of $T$. polium, which are similar to other researchers in Iran and elsewhere in the world, which can be compared to the cause of the climatic and geographical conditions. The results of the study of antibacterial effects of T. polium plant in Sistan and Baluchistan province showed that the plant's antiseptic effect on gram-negative bacteria of $K$. pneumoniae with a growth inhibitory diameter of $15 \mathrm{~mm}$ was. In a recent study, the antihypertensive effect of the T. polium was experimentally approved (18). Furthermore a recent study detected that $T$. polium significantly reduced cholesterol and triglyceride levels in experimental mice (19).

Klebsiella pneumoniae is a gram-negative bacterium due to a range of diseases such as pneumonia, urinary tract infections, septicemia, soft tissue infections, bacterial meningitis and hepatitis. It is also the cause of hospital infections and acquired diseases. K. pneumoniae in the hospital, where colonization is directly related to the length of hospitalization, dramatically increases. Long stay in a hospital increases the percentage of the presence of K. pneumoniae on the skin of patients and even hospital staff (20).

In the present study, 120 isolates of K. pneumoniae in hospitalized patients studied and the highest number was related to ICUs with 23 cases (19.2\%). Previously, a study in Iran, on 200 clinical isolates of K. pneumoniae, which were collected from the ICU, urology, respiratory and surgical departments during a year, showed the highest frequency was isolated from the ICU (21), which is consistent with our study.

Our results showed that the level of resistance to thirdgeneration cephalosporin is relatively high among clinical isolates of K. pneumoniae in Zahedan, since due to the lack of effective antibiotics for the treatment of these pathogens, this level of resistance can have many problems in the future. Finally, it should be noted that widespread control of ESBLs is difficult and may only be possible through the implementation of health and medical programs considering high cost. Therefore, the primary frequency control of the organisms producing ESBLs in a hospital or special department of the hospital is very important.

\section{Conclusion}

The results indicated the high inhibitory and microbial strength of T. polium. The antibacterial effects of T. polium can be attributed to the compounds of a-pinene and Linalool, which have been approved the antibacterial effects of these compounds. Therefore, due to the antibacterial effects of T. polium extract in comparison with the thirdgeneration cephalosporin antigens, this antibacterial agent can be used as a combination with antibacterial effects of natural origin. Our results showed that the level of resistance to third-generation cephalosporin among the clinical isolates of $K$. pneumoniae in Zahedan was relatively high and due to the lack of effective antibiotics for the treatment of these pathogens, this resistance could be a major problem in the future in treatment of infections.

\section{Authors' Contribution}

Study concept and design: JD, FRZ and RGDM. Dara analysis: RGDM and FRZ. Interpretation of data: FRZ and JD. Draft of the manuscript: JD and RGDM . Final revision: RGDM and FRZ. Study supervision: RGDM and FRZ.

Conflicts of interest

The authors declare no conflict of interest.

\section{Ethical considerations}

Ethical issues (including plagiarism, data fabrication, double publication) have been completely observed by the authors.

\section{Funding/Support}

This research with approved code $\# 1099706040002$ was funded by the Deputy Research and Vice-Chancellor of Research and financial affairs and research associate of Islamic Azad University of Zahedan 
and collaborated with the infectious-tropical Research Center of Zahedan University of Medical Sciences in Bu Ali hospital.

\section{References}

1. Manoharan A, Sugumar M, Kumar A, Jose H, Mathai D, ICMRESBL study group. Phenotypic \& molecular characterization of AmpC $\beta$-lactamases among Escherichia coli, Klebsiella spp. \& Enterobacter spp. from five Indian Medical Centers. Indian J Med Res. 2012;135:359.

2. Shanthi J, Balagurunathan R. Characterisation of heteroresistant subcolonies for MBL, AmpC genes in Klebsiella pneumoniae and Acinetobacter baumannii. Indian J Med Microbiol. 2014;32:210-1. doi: 10.4103/0255-0857.129869

3. Mohammad M, Salari H, Farahmand A. Anti-bacterial effects of the essential oil of Teucrium polium L. on human pathogenic bacteria. Iranian J Med Microbiol. 2013 Sep 15;7:1-7.

4. Jalalpoor S. Antibiotic resistant pattern in ESBLs producer Klebsiella pneumoniae strains isolated of hospitalized and out patients acquired urinary tract infection. J Isfahan Med Sch 2011;29(142).

5. Manoharan A, Sugumar M, Kumar A, Jose H, Mathai D, ICMRESBL study group. Phenotypic \& molecular characterization of AmpC $\beta$-lactamases among Escherichia coli, Klebsiella spp. \& Enterobacter spp. from five Indian Medical Centers. Indian J Med Res. 2012 ; 135: 359-364.

6. Performance standards for antimicrobial susceptibility testing: Twentieth informational supplement. CLSI document M100-S20. Wayne PA: Clinical Laboratory Standard Institute (CLSI); 2010.

7. Ahmed $\mathrm{OB}$, Omar AO, Asghar AH, Elhassan MM, AlMunawwarah AM. Prevalence of TEM, SHV and CTX-M genes in Escherichia coli and Klebsiella spp urinary isolates from Sudan with confirmed ESBL phenotype. Life Sci J. 2013;10:191-5.

8. Sękowska A, Gospodarek E, Kamińska D. Antimicrobial susceptibility and genetic similarity of ESBL-positive Klebsiella pneumoniae strains. Arch Med Sci. 2012;8(6):993-7. doi: 10.5114/aoms.2012.32404.

9. Baher Z, Mirza M. Volatile constituents of Teucrium flavum L. from Iran. J Essen Oil Res. 2003;15:106-107. doi: 10.1080/10412905.2003.9712082

10. Jaimand K, Rezaee MB, Soltanipoor MA, Mozaffarian V.
Volatile constituents of Teucrium stocksianum Boiss. ssp. stocksianum from Iran. J Essen Oil Res. 2006 Sep 1;18:476-7. doi: 10.1080/10412905.2006.9699145

11. Javidnia, K. and R. Miri, Composition of the Essential Oil of Teucrium orientate L. ssp. orientate from Iran. J Essen Oil Res. 2003; 15:118-119 doi: 10.1080/10412905.2003.9712086.

12. Javidnia K. Miri R, Khosravi A. Composition of the essential oil of Teucrium persicum Boiss. from Iran. J Essen Oil Res. 2007; 19 :430-432. doi: 10.1080/10412905.2007.9699944

13. Amiri H. Chemical Composition of essential oil of Teucrium orientale L. subsp. taylori (Boiss.) Rech. f.. J Med Plants. 2008; $4: 100-104$

14. Flamini G, Cioni PL, Morelli I, Maccioni S, Monti G. Composition of the essential oil of Teucrium fruticans $\mathrm{L}$. from the Maremma Regional Park (Tuscany, Italy). Flavour Fragrance J. 2001;16:367-9. doi: 10.1002/ffj.1014

15. Cozzani S, Muselli A, Desjobert JM, Bernardini AF, Tomi F, Casanova J. Chemical composition of essential oil of Teucrium polium subsp. capitatum (L.) from Corsica. Flavour Fragrance J. 2005 Jul;20:436-41. doi: 10.1002/ffj.1463

16. Aburjai T, Hudaib M, Cavrini V. Composition of the essential oil from Jordanian germander (Teucrium polium L.). J Essen oil Res. 2006 Jan 1;18:97-9. doi: 10.1080/10412905.2006.9699398

17. Cakir A, Duru ME, Harmandar M, Ciriminna $R$, Passannanti S. Volatile constituents of Teucrium polium L. from Turkey. J Essen oil Res. 1998 Jan 1;10:113-5. doi: 10.1080/10412905.1998.9700855

18. Suleiman MS, Abdul-Ghani AS, Al-Khalil S, Amin R. Effect of Teucrium polium boiled leaf extract on intestinal motility and blood pressure. J Ethnopharmacol. 1988 Jan 1;22:111-6. doi: 10.1016/0378-8741(88)90236-x

19. Rasekh HR, Khoshnood-Mansourkhani MJ, Kamalinejad M. Hypolipidemic effects of Teucrium polium in rats. Fitoterapia. 2001 Dec 1;72:937-9. doi: 10.1016/s0367-326x(01)00348-3

20. Pourali Sheshblouki G, Mardaneh J. Characterization of blaCTX gene and cross-resistance in Klebsiella pneumoniae isolated from hospitalized patients. J Birjand Univ Med Sci. 2016; 23:56-66

21. Nasehi L, Shah CF, Nikbin VA, Nematzadeh SH. PER, CTX-M, TEM and SHV Beta-lactamases in clinical isolates of Klebsiella pneumoniae isolated from Tehran, Iran. Iranian J Basic Med Sci. 2010;13:111-8. doi: 10.22038/JBBMS.2010.5096 